\title{
Sparse regularization methods in ultrafast ultrasound imaging
}

Adrien Besson ${ }^{1}$, Rafael E. Carrillo ${ }^{1}$, Miaomiao Zhang ${ }^{2}$, Denis Friboulet $^{2}$, Olivier Bernard ${ }^{2}$, Yves Wiaux ${ }^{3}$ and Jean-Philippe Thiran $^{1}$

${ }^{1}$ Signal Processing Laboratory (LTS5)

École Polytechnique Fédérale de Lausanne, Switzerland

${ }^{2}$ CREATIS

University of Lyon, France

${ }^{3}$ Institute of Sensors, Signals and Systems

Heriot Watt University, Scotland

European Signal Processing Conference 2016, August 30, 2016 


\section{Outline}

Ultrafast ultrasound imaging

Principle

Spatial-based approaches

Fourier-based approaches

Sparse regularization for ultrasound imaging

General framework

The measurement model

Sparsifying model and reconstruction algorithm

Applications of the proposed approach

Sparse regularization for image quality enhancement

Compressed beamforming

Conclusion 


\section{Outline}

Ultrafast ultrasound imaging

Principle

Spatial-based approaches

Fourier-based approaches

Sparse regularization for ultrasound imaging

General framework

The measurement model

Sparsifying model and reconstruction algorithm

Applications of the proposed approach

Sparse regularization for image quality enhancement

Compressed beamforming

Conclusion 


\section{Ultrafast Ultrasound Imaging \\ Principle}

Ultrafast ultrasound imaging 


\section{Ultrafast Ultrasound Imaging \\ Principle}

Ultrafast ultrasound imaging

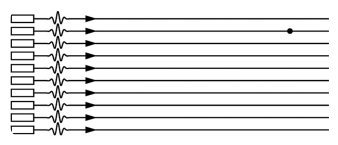

(a) Transmit 


\section{Ultrafast Ultrasound Imaging Principle}

Ultrafast ultrasound imaging

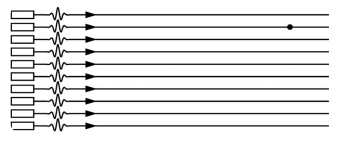

(a) Transmit

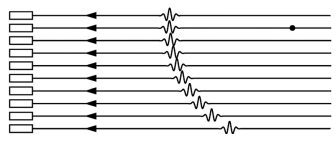

(b) Receive (Source: David et al., 2015) 


\section{Ultrafast Ultrasound Imaging Principle}

Ultrafast ultrasound imaging

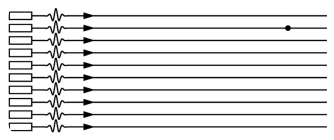

(a) Transmit

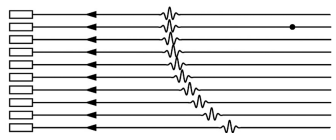

(b) Receive (Source: David et al., 2015)

Image reconstruction - Beamforming 


\section{Ultrafast Ultrasound Imaging Principle}

Ultrafast ultrasound imaging

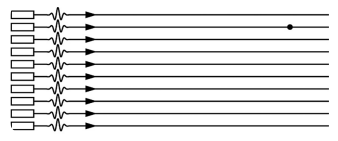

(a) Transmit

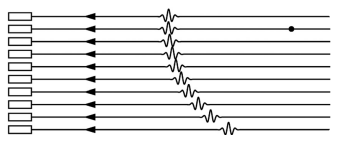

(b) Receive (Source: David et al., 2015)

Image reconstruction - Beamforming

- Infer the reflectivity $s(x, z)$ from the received echoes $r\left(x_{i}, t\right)$ 


\section{Ultrafast Ultrasound Imaging Principle}

Ultrafast ultrasound imaging

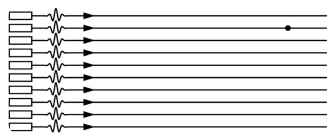

(a) Transmit

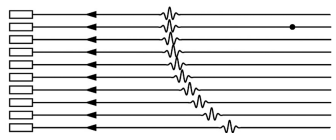

(b) Receive (Source: David et al., 2015)

Image reconstruction - Beamforming

- Infer the reflectivity $s(x, z)$ from the received echoes $r\left(x_{i}, t\right)$

- State of the art approaches 


\section{Ultrafast Ultrasound Imaging Principle}

Ultrafast ultrasound imaging

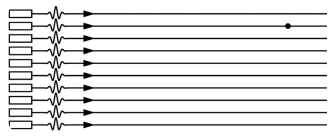

(a) Transmit

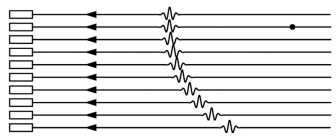

(b) Receive (Source: David et al., 2015)

Image reconstruction - Beamforming

- Infer the reflectivity $s(x, z)$ from the received echoes $r\left(x_{i}, t\right)$

- State of the art approaches

- Spatial-based approaches [Montaldo et al., 2009] 


\section{Ultrafast Ultrasound Imaging Principle}

Ultrafast ultrasound imaging

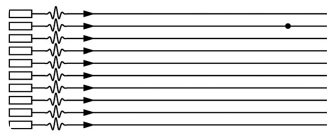

(a) Transmit

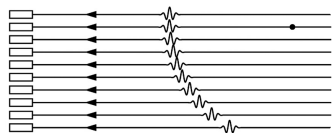

(b) Receive (Source: David et al., 2015)

Image reconstruction - Beamforming

- Infer the reflectivity $s(x, z)$ from the received echoes $r\left(x_{i}, t\right)$

- State of the art approaches

- Spatial-based approaches [Montaldo et al., 2009]

- Fourier-based approaches [Lu et al., 1997] [Garcia et al., 2013] [Bernard et al., 2014] 


\section{Ultrafast Ultrasound Imaging}

Spatial-based approaches

Delay-and-sum beamforming

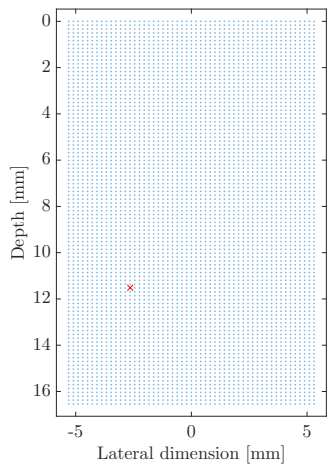

(a) 


\section{Ultrafast Ultrasound Imaging}

Spatial-based approaches

Delay-and-sum beamforming

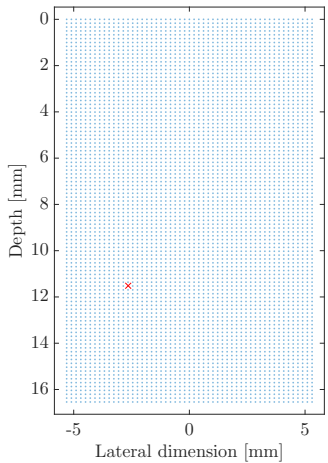

(a)

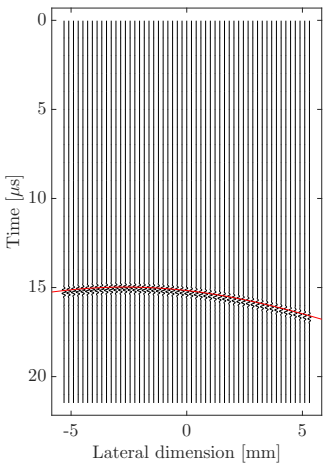

(b)

\section{EUSIPCO 2016}

$5 / 21$ 


\section{Ultrafast Ultrasound Imaging}

Spatial-based approaches

Delay-and-sum beamforming

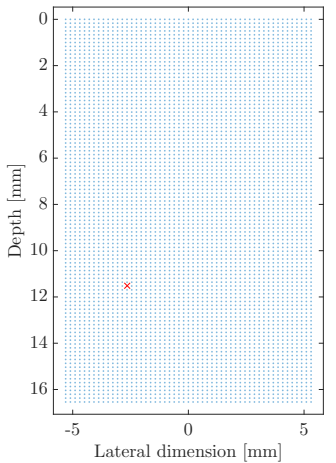

(a)

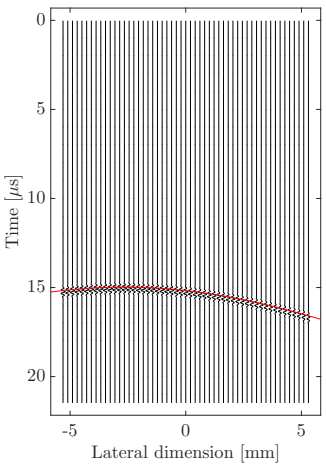

(b)

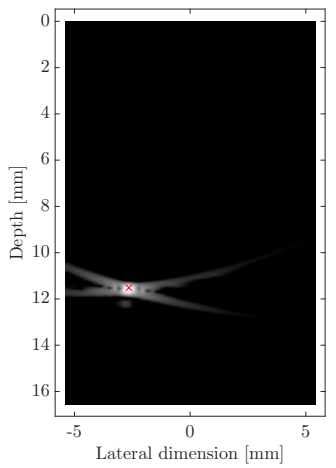

(c) 


\section{Ultrafast Ultrasound Imaging}

Fourier-based approaches

Principle 


\section{Ultrafast Ultrasound Imaging}

Fourier-based approaches

Principle

- Based on a remapping of the Fourier spectrum of the echoes in the space of the desired image 


\section{Ultrafast Ultrasound Imaging}

Fourier-based approaches

Principle

- Based on a remapping of the Fourier spectrum of the echoes in the space of the desired image 


\section{Ultrafast Ultrasound Imaging}

Fourier-based approaches

Principle

- Based on a remapping of the Fourier spectrum of the echoes in the space of the desired image

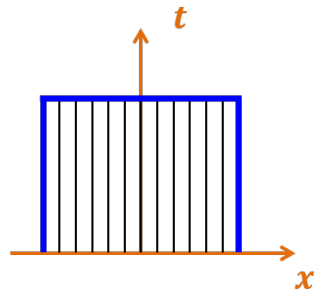

(a) Backscattered

echoes 


\section{Ultrafast Ultrasound Imaging}

Fourier-based approaches

Principle

- Based on a remapping of the Fourier spectrum of the echoes in the space of the desired image

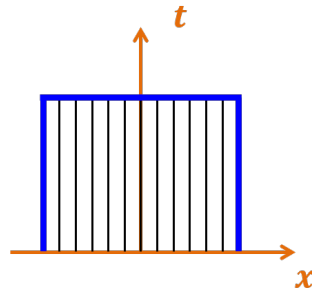

(a) Backscattered echoes

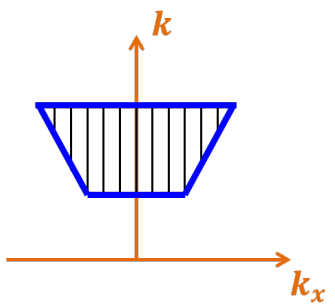

(b) Fourier spectrum 


\section{Ultrafast Ultrasound Imaging}

Fourier-based approaches

Principle

- Based on a remapping of the Fourier spectrum of the echoes in the space of the desired image

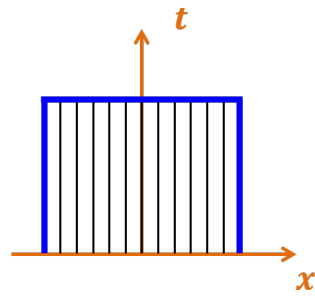

(a) Backscattered echoes

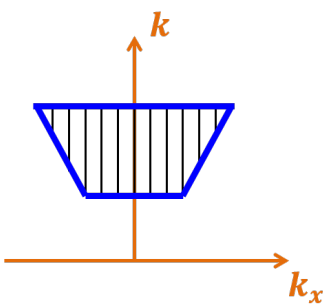

(b) Fourier spectrum

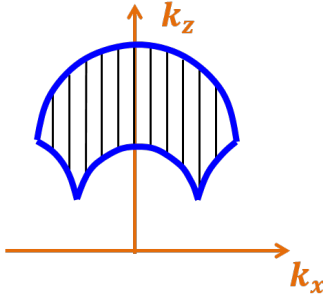

(c) Remapped Fourier spectrum 


\section{Ultrafast Ultrasound Imaging}

\section{Main drawbacks of the classical approaches}

Image reconstruction is an ill-posed problem

- The backscattered echoes do not necessarily carry all the information necessary to reconstruct images 


\section{Ultrafast Ultrasound Imaging}

\section{Main drawbacks of the classical approaches}

Image reconstruction is an ill-posed problem

- The backscattered echoes do not necessarily carry all the information necessary to reconstruct images

Interpolation on discrete grids creates artifacts 


\section{Ultrafast Ultrasound Imaging}

\section{Main drawbacks of the classical approaches}

Image reconstruction is an ill-posed problem

- The backscattered echoes do not necessarily carry all the information necessary to reconstruct images

Interpolation on discrete grids creates artifacts

- Interpolation of delays

- Interpolation of remapped Fourier spectrum 


\section{Ultrafast Ultrasound Imaging}

\section{Main drawbacks of the classical approaches}

Image reconstruction is an ill-posed problem

- The backscattered echoes do not necessarily carry all the information necessary to reconstruct images

Interpolation on discrete grids creates artifacts

- Interpolation of delays

- Interpolation of remapped Fourier spectrum

Classical methods are filtered-backprojection solutions of the inverse problem 


\section{Outline}

Ultrafast ultrasound imaging

Principle

Spatial-based approaches

Fourier-based approaches

Sparse regularization for ultrasound imaging

General framework

The measurement model

Sparsifying model and reconstruction algorithm

Applications of the proposed approach

Sparse regularization for image quality enhancement

Compressed beamforming

Conclusion

EUSIPCO 2016

$8 / 21$ 


\section{Sparse regularization for ultrasound imaging}

General framework

Sparse regularization as an alternative to filtered-backprojection 


\section{Sparse regularization for ultrasound imaging}

General framework

Sparse regularization as an alternative to filtered-backprojection

- Use sparse regularization methods to solve the inverse problem posed by the image reconstruction 


\section{Sparse regularization for ultrasound imaging}

\section{General framework}

Sparse regularization as an alternative to filtered-backprojection

- Use sparse regularization methods to solve the inverse problem posed by the image reconstruction

- Two pillars: 


\section{Sparse regularization for ultrasound imaging}

\section{General framework}

Sparse regularization as an alternative to filtered-backprojection

- Use sparse regularization methods to solve the inverse problem posed by the image reconstruction

- Two pillars:

- The ability to express acquisition as a linear inverse problem $\boldsymbol{r}=\mathrm{H} \boldsymbol{s}+\boldsymbol{n}$ with $\boldsymbol{r} \in \mathbb{R}^{M}$ the element raw data and $\boldsymbol{s} \in \mathbb{R}^{N}$ the desired image 


\section{Sparse regularization for ultrasound imaging}

\section{General framework}

Sparse regularization as an alternative to filtered-backprojection

- Use sparse regularization methods to solve the inverse problem posed by the image reconstruction

- Two pillars:

- The ability to express acquisition as a linear inverse problem $\boldsymbol{r}=\mathrm{H} \boldsymbol{s}+\boldsymbol{n}$ with $\boldsymbol{r} \in \mathbb{R}^{M}$ the element raw data and $\boldsymbol{s} \in \mathbb{R}^{N}$ the desired image

- The sparsity of ultrasound images in a given model $\Psi$ 


\section{Sparse regularization for ultrasound imaging}

\section{General framework}

Sparse regularization as an alternative to filtered-backprojection

- Use sparse regularization methods to solve the inverse problem posed by the image reconstruction

- Two pillars:

- The ability to express acquisition as a linear inverse problem $\boldsymbol{r}=\mathrm{H} \boldsymbol{s}+\boldsymbol{n}$ with $\boldsymbol{r} \in \mathbb{R}^{M}$ the element raw data and $\boldsymbol{s} \in \mathbb{R}^{N}$ the desired image

- The sparsity of ultrasound images in a given model $\Psi$

Image reconstruction An analysis-based problem is solved $\left(\ell_{1}\right.$-minimization)

$$
\min _{\overline{\boldsymbol{s}} \in \mathbb{C}^{N}}\left\|\Psi^{H} \overline{\boldsymbol{s}}\right\|_{1} \text { subject to }\|\boldsymbol{r}-\mathrm{H} \overline{\boldsymbol{s}}\|_{2} \leq \epsilon
$$




\section{Sparse regularization for ultrasound imaging}

Measurement model

Spatial-based approach 
Sparse regularization for ultrasound imaging

Measurement model

Spatial-based approach

- In the continuous domain:

$$
r\left(x_{i}, t\right)=\iint_{(x, z) \in \Omega\left(x_{i}, t\right)} s(x, z) d x d z
$$

$\Omega\left(x_{i}, t\right)$ propagation delay curve 
Sparse regularization for ultrasound imaging

Measurement model

Spatial-based approach

- In the continuous domain:

$$
r\left(x_{i}, t\right)=\iint_{(x, z) \in \Omega\left(x_{i}, t\right)} s(x, z) d x d z
$$

$\Omega\left(x_{i}, t\right)$ propagation delay curve

- Discretization of the integral leads to $\boldsymbol{r}=\mathrm{H}_{\mathrm{S}} \boldsymbol{s}+\boldsymbol{n}$ 
Sparse regularization for ultrasound imaging

Measurement model

Spatial-based approach

- In the continuous domain:

$$
r\left(x_{i}, t\right)=\iint_{(x, z) \in \Omega\left(x_{i}, t\right)} s(x, z) d x d z
$$

$\Omega\left(x_{i}, t\right)$ propagation delay curve

- Discretization of the integral leads to $\boldsymbol{r}=\mathrm{H}_{\mathrm{S}} \boldsymbol{s}+\boldsymbol{n}$

Fourier-based approach [Besson et al, 2016] 
Sparse regularization for ultrasound imaging

Measurement model

Spatial-based approach

- In the continuous domain:

$$
r\left(x_{i}, t\right)=\iint_{(x, z) \in \Omega\left(x_{i}, t\right)} s(x, z) d x d z
$$

$\Omega\left(x_{i}, t\right)$ propagation delay curve

- Discretization of the integral leads to $\boldsymbol{r}=\mathrm{H}_{\mathrm{S}} \boldsymbol{s}+\boldsymbol{n}$

Fourier-based approach [Besson et al., 2016]

$$
\boldsymbol{r}=\mathrm{F}_{\mathrm{R}}^{\mathrm{H}} \mathrm{GF}_{\mathrm{S}} \boldsymbol{s}+\boldsymbol{n}
$$

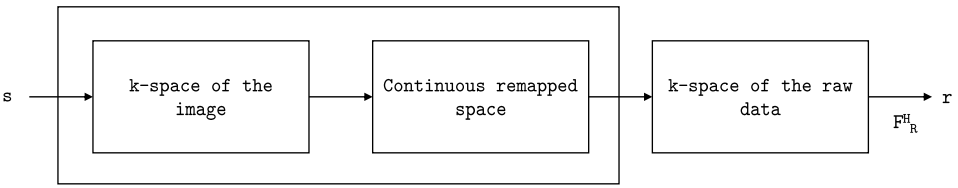

$\mathrm{GF}_{\mathrm{S}}$ 


\section{Sparse regularization for ultrasound imaging}

Sparsifying model and reconstruction algorithm

Sparsifying model 


\section{Sparse regularization for ultrasound imaging}

Sparsifying model and reconstruction algorithm

Sparsifying model

- Several models already studied in the literature: Wave Atom frame, Fourier basis, Dirac basis, Wavelet-based models 


\section{Sparse regularization for ultrasound imaging}

Sparsifying model and reconstruction algorithm

Sparsifying model

- Several models already studied in the literature: Wave Atom frame, Fourier basis, Dirac basis, Wavelet-based models

- Sparsity averaging model (SA) $\Psi$ used [Carrillo et al, 2013] : 


\section{Sparse regularization for ultrasound imaging}

Sparsifying model and reconstruction algorithm

Sparsifying model

- Several models already studied in the literature: Wave Atom frame, Fourier basis, Dirac basis, Wavelet-based models

- Sparsity averaging model (SA) $\Psi$ used [Carillo et al, 2013] :

- Concatenation of wavelet bases: $\Psi=\frac{1}{\sqrt{q}}\left[\Psi_{1}, \ldots, \Psi_{q}\right]$ 


\section{Sparse regularization for ultrasound imaging}

Sparsifying model and reconstruction algorithm

Sparsifying model

- Several models already studied in the literature: Wave Atom frame, Fourier basis, Dirac basis, Wavelet-based models

- Sparsity averaging model (SA) $\Psi$ used [Carillo et al, 2013] :

- Concatenation of wavelet bases: $\Psi=\frac{1}{\sqrt{q}}\left[\Psi_{1}, \ldots, \Psi_{q}\right]$

- In the study: $q=8$, Daubechies wavelet as mother function 
Sparse regularization for ultrasound imaging

Sparsifying model and reconstruction algorithm

Sparsifying model

- Several models already studied in the literature: Wave Atom frame, Fourier basis, Dirac basis, Wavelet-based models

- Sparsity averaging model $(S A) \Psi$ used [Carillo et al, 2013] :

- Concatenation of wavelet bases: $\Psi=\frac{1}{\sqrt{q}}\left[\Psi_{1}, \ldots, \Psi_{q}\right]$

- In the study: $q=8$, Daubechies wavelet as mother function

Image reconstruction problem

- Analysis-based problem:

$$
\min _{\overline{\boldsymbol{s}} \in \mathbb{C}^{N}}\left\|\Psi^{H} \overline{\boldsymbol{s}}\right\|_{1} \text { subject to }\|\boldsymbol{r}-\mathrm{H} \overline{\boldsymbol{s}}\|_{2} \leq \epsilon
$$

with $\Psi$ the SA model and $\mathrm{H}$ Spatial-based or Fourier-based reconstruction model

- Alternating Direction of Minimizers (ADMM) used to solve the problem 


\section{Outline}

Ultrafast ultrasound imaging

Principle

Spatial-based approaches

Fourier-based approaches

Sparse regularization for ultrasound imaging

General framework

The measurement model

Sparsifying model and reconstruction algorithm

Applications of the proposed approach

Sparse regularization for image quality enhancement

Compressed beamforming

Conclusion 


\section{Applications of the proposed approach}

Sparse regularization for image quality enhancement

Problem \& Objective 


\section{Applications of the proposed approach}

Sparse regularization for image quality enhancement

Problem \& Objective

- Image reconstruction poses an ill-posed problem

- Discretization of the measurement models leads to inaccuracies [Carrillo et al., 2015]

- Use sparse regularization to enhance image quality 


\section{Applications of the proposed approach}

\section{Sparse regularization for image quality enhancement}

Problem \& Objective

- Image reconstruction poses an ill-posed problem

- Discretization of the measurement models leads to inaccuracies [Carrillo et al., 2015]

- Use sparse regularization to enhance image quality

Numerical study of the contrast

- 8-mm anechoic inclusion positioned at $4 \mathrm{~cm}$ embedded in a medium with high density of scatterers

- Insonification with 1 plane wave

- Reconstruction with classical approaches and sparse regularization 


\section{Applications of the proposed approach}

Sparse regularization for image quality enhancement

B-mode images of the anechoic inclusion

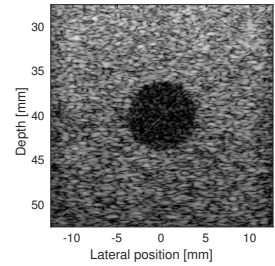

(a) Classical

$\mathrm{CR}=5.59 \mathrm{~dB}$

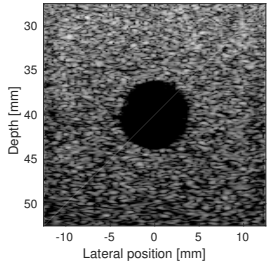

(b) Sparse spatial

$\mathrm{CR}=9.10 \mathrm{~dB}$

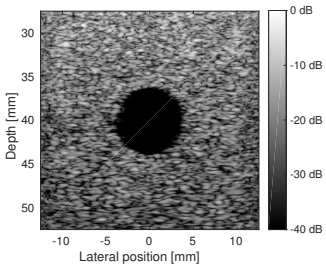

(c) Sparse Fourier

$\mathrm{CR}=9.62 \mathrm{~dB}$ 


\section{Applications of the proposed approach}

Compressed beamforming

Problem \& Objective 


\section{Applications of the proposed approach}

Compressed beamforming

Problem \& Objective

- Acquisition with few transducer elements 


\section{Applications of the proposed approach}

Compressed beamforming

Problem \& Objective

- Acquisition with few transducer elements

- Creation of a new measurement vector: $\boldsymbol{r}_{\boldsymbol{u}}=\mathrm{P} \boldsymbol{r}$ with

$\mathrm{P} \in \mathbb{R}^{R \times M}$ and $R \ll M$ 


\section{Applications of the proposed approach}

Compressed beamforming

Problem \& Objective

- Acquisition with few transducer elements

- Creation of a new measurement vector: $\boldsymbol{r}_{\boldsymbol{u}}=\mathrm{P} \boldsymbol{r}$ with

$\mathrm{P} \in \mathbb{R}^{R \times M}$ and $R \ll M$

- Retrieve high quality images from this undersampled measurement vector [Besson et al., 2016]

$$
\min _{\overline{\boldsymbol{s}} \in \mathbb{C}^{N}}\left\|\Psi^{H} \overline{\boldsymbol{s}}\right\|_{1} \text { subject to }\left\|\boldsymbol{r}_{\boldsymbol{u}}-\mathrm{H}_{\mathrm{u}} \overline{\boldsymbol{s}}\right\|_{2} \leq \epsilon
$$

with $\mathrm{H}_{\mathrm{u}}=\mathrm{PH} \in \mathbb{R}^{P \times N}$ 


\section{Applications of the proposed approach}

Compressed beamforming

Experiments

- Acquisition of an in-vivo carotid with a Verasonics US system

- 1 PW insonification with a 128 transducer-elements probe

- Random selection of 30 transducer elements in receive

- Reconstruction with the sparse regularization approach 


\section{Applications of the proposed approach}

Compressed beamforming

B-mode images obtained with 30 transducer elements

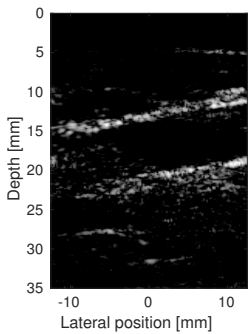

(a) Fourier

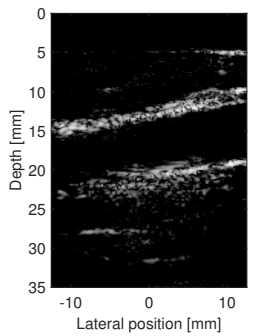

(b) Spatial

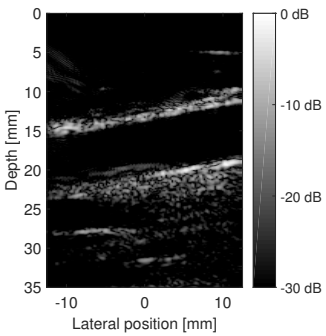

(c) Reference 


\section{Applications of the proposed approach}

Compressed beamforming

Peak-signal-to-noise-ratio against number of transducers

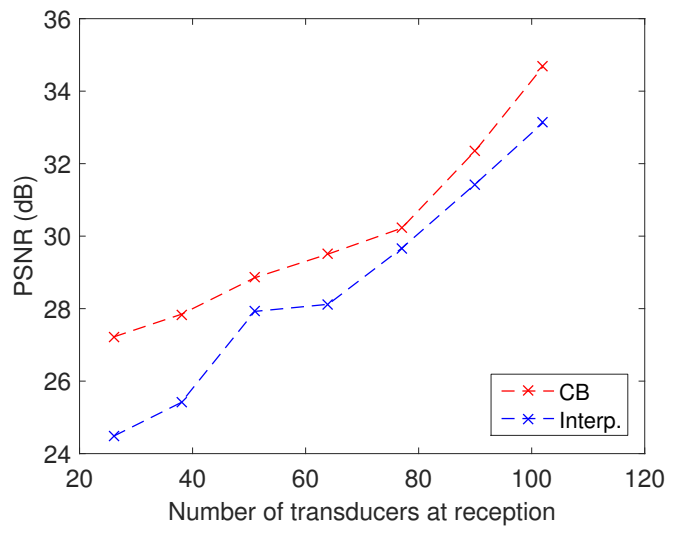




\section{Outline}

Ultrafast ultrasound imaging

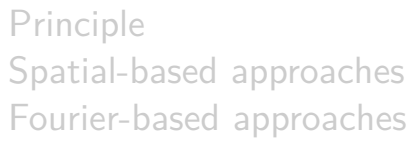

Sparse regularization for ultrasound imaging

General framework

The measurement model

Sparsifying model and reconstruction algorithm

Applications of the proposed approach

Sparse regularization for image quality enhancement

Compressed beamforming

Conclusion

EUSIPCO 2016

$19 / 21$ 


\section{Conclusion \& Perspectives}

Framework for sparse regularization in US imaging

- Measurement model for Fourier-based and spatial-based approaches

- Sparsity Averaging model as a sparsifying model

- Image reconstruction algorithm 


\section{Conclusion \& Perspectives}

Framework for sparse regularization in US imaging

- Measurement model for Fourier-based and spatial-based approaches

- Sparsity Averaging model as a sparsifying model

- Image reconstruction algorithm

Applications

- Image quality enhancement (contrast)

- Compressed beamforming 


\section{Conclusion \& Perspectives}

Framework for sparse regularization in US imaging

- Measurement model for Fourier-based and spatial-based approaches

- Sparsity Averaging model as a sparsifying model

- Image reconstruction algorithm

Applications

- Image quality enhancement (contrast)

- Compressed beamforming

Perspectives

- Extension to 3D imaging

- More robust reconstruction algorithms which take into account spatially variable point spread functions 


\section{Thank you!}

EUSIPCO 2016

$21 / 21$ 